\title{
Postural tachycardia syndrome, the debate continues...
}

\author{
Christopher Gibbons
}

Received: 10 October 2014/ Accepted: 29 October 2014/Published online: 13 November 2014

(C) Springer-Verlag Berlin Heidelberg 2014

Postural tachycardia syndrome (POTS) is a relatively recent addition to the scientific literature. A simple perusal of PubMed will determine that Rosen and Cryer described POTS for the first time in 1982. [1] Since that initial description, over 500 publications have been associated with POTS, over 470 of which have been published since the year 2000. The rapid escalation in publication frequency could suggest a possible epidemic of POTS, with vast numbers of individuals afflicted.

However, more detailed review of the scientific literature will reveal descriptions of similar entities such as irritable heart syndrome, effort syndrome, orthostatic intolerance, mitral valve prolapse syndrome, neurocirulatory asthenia, orthostatic tachycardia, vasoregulatory asthenia, hyperadrenergic orthostatic tachycardia, hyperdynamic beta-adrenergic state, and a host of other possible entities [2, 3]. Into this quagmire we have placed a new term, 'Postural tachycardia syndrome', which defines the syndrome with a simple, clear, and clinically relevant change in heart rate [4]. POTS: A postural increase in standing heart rate of 30 beats per minutes, without accompanying orthostatic hypotension, or a standing heart rate of 120 beats per minute (there is a caveat that individuals ages 12-19 may require an increase in heart rate of $>40$ beats per minute) [4]. Despite the simple definition of POTS, there is an enormous clinical heterogeneity that thwarts all attempts at standardization of scientific nomenclature. Various modifiers have now been appended to POTS such as 'high-flow, low-flow, dipping, adrenergic, neuropathic' and others. These terms, although meant to

C. Gibbons $(\square)$

Beth Israel Deaconess Medical Center, 1 Deaconess Rd,

Palmer 111, Boston, MA 02215, USA

e-mail: cgibbons@bidmc.harvard.edu provide clarity, have resulted in significant confusion among practitioners and investigators about the appropriate classification of people with a postural tachycardia and associated orthostatic symptoms.

The article entitled "A prospective study of excessive postural heart rate change on head-up tilt." by Corkal and Kimpinski highlights the ongoing challenges associated with making a clear diagnosis of POTS. In this study, the authors performed autonomic testing, including supine and upright tilted heart rates in a large number of healthy individuals and found a postural tachycardia of $>30$ beats per minute in 26 people (age $24 \pm 7$ years). At baseline, these individuals did not have orthostatic symptoms using standardized questionnaires, and no other evidence of autonomic dysfunction on formal testing. They retested these same individuals 1 year later. For most individuals, they continued to have a postural tachycardia. However, they did not develop symptoms of orthostatic intolerance and had no other abnormalities on autonomic testing.

The individuals in this study were selected from a group of 170 healthy subjects (ages 13-80) recruited for development of a normative database. These data suggest that at least $15 \%$ of the population studied has a postural tachycardia using current definitions. However, the subjects reported in the current study were predominantly younger and made up a substantial proportion of healthy subjects in the $<40$ age group. These results suggest that a postural tachycardia of $>30$ beats per minute is commonly seen in individuals $<40$ years of age and is part of the expected variance in the population. A postural tachycardia alone does not predispose subjects to development of POTS, or of development of autonomic dysfunction. These findings challenge the utility of a strict diagnostic threshold (an orthostatic heart rate change of 30 beats per minute) that overlaps too highly with the normal variance in the 
population. The study by Corkal and Kimpinsky supports the need for age specific normative values in the definition of a postural tachycardia, without which our understanding of the pathophysiology of this disease will continue to suffer by inclusion of inappropriate individuals in studies of POTS.

\section{References}

1. Rosen SG, Cryer PE (1982) Postural tachycardia syndrome. Reversal of sympathetic hyperresponsiveness and clinical improvement during sodium loading. Am J Med 72:847-850
2. Wooley CF (1976) Where are the diseases of yesteryear? DaCosta's syndrome, soldiers heart, the effort syndrome, neurocirculatory asthenia-and the mitral valve prolapse syndrome. Circulation 53:749-751

3. Streeten DH (1999) Orthostatic intolerance. A historical introduction to the pathophysiological mechanisms. Am J Med Sci 317:78-87

4. Freeman R, Wieling W, Axelrod FB et al (2011) Consensus statement on the definition of orthostatic hypotension, neurally mediated syncope and the postural tachycardia syndrome. Clin Auton Res 21:69-72 